\title{
Waves in Plasmas: Highlights from the Past and Present*
}

\author{
Thomas H. Stix \\ Princeton Plasma Physics Laboratory, Princeton University \\ Princeton, New Jersey 08543
}

PPPL -2680

DE90 008004

\begin{abstract}
To illustrate the development of some fundamental concepts in plasma waves, a number of experimental observations, going back over half a century, are reviewed. Particular attention is paid to the phenomena of dispersion, collisionfree damping, ray trajectories, amplitude transport, plasma wave echos, finite-Larmor-radius and cyclotron and cyclotron-harmonic effects, nonlocal response, and mode conversion. Also to the straight-trajectory approximation and two-level phase mixing. And to quasilinear diffusion and its relation to radiofrequency heating, current drive and induced neoclassical transport, and to stochasticity and superadiabaticity. One notes not only the constructive interplay between experiment and theory but also that major advances have come from each of the many disciplines that invoke plasma physics as a tool including radio communication, astrophysics, controlled fusion, space physics, and basic research.
\end{abstract}

National Science Foundation Lecture commemorating the award of the 25,000th NSF Graduate Fellowship, presented on 13 November 1989 at the Thirty-First Annual meeting of the American Physical Society's Division of Plasma Physics. Portions of the talk were presented earlier, at the International Conference on Plasma Physics, Lausanne, Switzerland, Summer 1984.

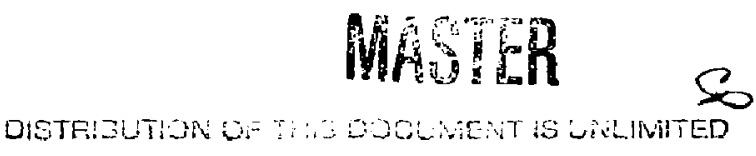




\section{Dispersion}

A major reward in the study of plasma physics is the very richness of the subject: even pictured just as a conducting fluid, the plasma medium can still be anisotropic, inhomogeneous, lossy or almost lossfree, and dispersive. The word "dispersive" usually inplies that the dielectric properties of the medium depend on the frequency of the wave, hence the velocity of the wave through the medium also depends upon the wave frequency. Perhaps the first property documented for plasma waves concerned their dispersivc character. The observations are described in colorful fashion by Barkhausen ${ }^{1}$ in 1919. He relates that audio amplifiers were used extensively on both sides of the Front in World War I, to eavesdrop on enemy telephone conversations. In a simple circuit, shown in Fig. 1, wires from two ground points, possibly a few hundred melers apart, are connected to an amplifier ( $V$ ) which feeds a telephone receiver ( $T$ ). The World War 1 military telephone systems - perhaps lorig single lines with ground retum - were evidently quite vulnerable to this early form of electronic surveillance. But, Barkhausen notes, sometimes one heard a quite remarkable whistling tone. At the Front, it was said that one could hear "flying grenades," and at times, especially in the forenoon on warm days in May and June, the phenomenon was so strong and so frequent as to make the eavesdropping impossible. Attempting to describe the sound in print, Barkhausen suggests it would be something like "piou." More physically, he sketches in Fig. 2 the descending tone, running down the whole audible scale with the lowest tones fading away, the entire event lasting about a second.

Although the same phenomenon was seen Fig. 2. Sketch or a whistlei tone. From Barkhausen (1919).

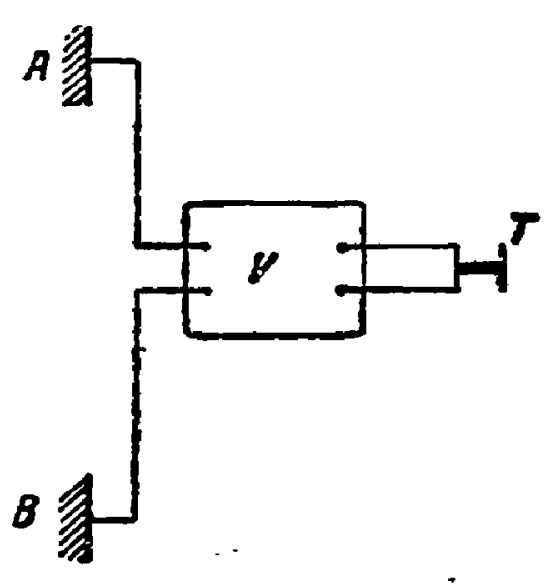

Fig. 1. Circuit for monitoring enemy telephone conversations in World War $l, V$ denotes an audio amplifier (Verstarker), $T$ a telephone. From Barkhausen (1919).

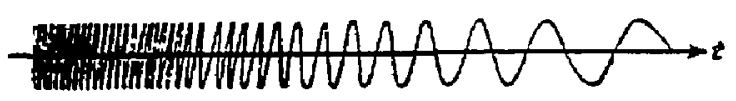

and discussed in the literature over the succeeding years, it was not until 1935 that the correct explanation was offered by Eckersley. ${ }^{2}$ In a letter 
to Nature, Eckersley reproduced, Fig. 3, some 1933 data taken by Burton and Boardman. ${ }^{3}$ On the basis of magneto-ionic theory (which we would now call "cold-plasma wave theory"), Eckersley surmises that the effect is due to the dispersion of a pulse in the ionosphere. Eckersley suggests that the pulse could arise from a flash of lightning, producing waves through the jonosphere which are then reflected at the polar regions. He states (without proof) that magneto-ionic theory would predict that the observed frequency at time $t$ after the beginning of the pulse is proportional to $1 / t^{2}$.

The details were finally filled in by Storey, ${ }^{4}$ in 1953. Storey's simplified dispersion selation describing this whistler mode is just

$$
\frac{\omega}{\mathbf{k}}=\overrightarrow{\mathrm{k}} \cdot \frac{\overrightarrow{\mathrm{B}}_{\mathrm{o}}}{\mathbf{B}_{\mathrm{o}}} \frac{\omega_{\mathrm{cc}} \mathrm{c}^{2}}{\omega_{\mathrm{p} \mathrm{e}}^{2}} .
$$

A little calculation will show that the group velocity is confined to a narrow cone around $\vec{B}_{0}$, and that it is proportional to $\omega^{1 / 2}$. The observed frequency, in agreement with Eckersley's statement, then descends just as $\mathrm{t}^{-2}$. Storey's model for the whistlers is shown in Fig. 4, illustrating the ray paths for "short" and "long whistlers.

While this 1953 picture of whistler ray paths is one with which we are all now familiar, it is interesting to see how long it took for this degree of understanding to come about. The problem with which many excellent radio physicists wrestled during the late 1920's and the early 1930's was the question of propagation paths through the ionosphere. Most attention was paid to the case of

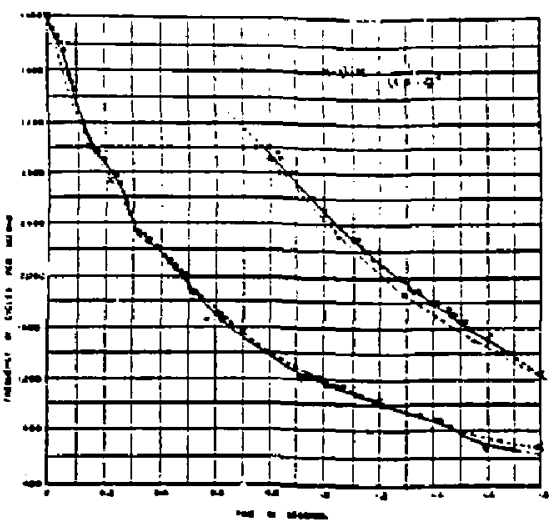

Fig. 3. Pair of whistler signals. Frequency versus time. Data from Burton and Boardman (1933), analyzed by Eckersley (1935).

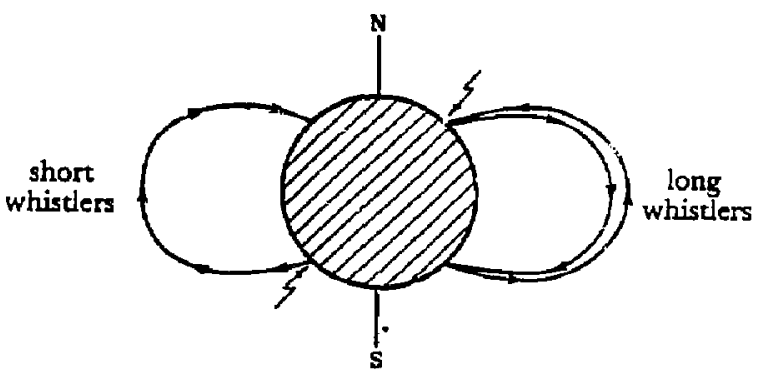

Fig. 4. Ray paths for whistler propagation through the magnetosphere. From Storey (1953). 
vertical incidence because looking at the echoes of an upward-directed radio pulse was a very popular experiment in the early 1930's. However this case is a special one, and the more general case of oblique incidence upon the ionosphere, taking into account the earth's magnetic field which generally has an inconvenient vector orientation, led to some puzzling results. An analysis in 1930 by the Russian radiophysicist Schekulin, 5 using Snells' Law and the magneto-ionic dispersion relation evaluated at different heights in the ionosphere, gave apparent propagation paths for oblique incidence as in Fig. 5. As noted in 1938 by H. G. Booker, ${ }^{6}$ Schekulin "does not, however, offer any convincing interpretation of such a curious result." It wás Booker, eight years after Schekulin's publication, who explained the anomaly. Schekulin's calculations were correct, but the arrows that he had connected together in his diagram were the phase-velocity vectori, not the group velocity. Putting in the group-velocity vectors and connecting them together then produced credible propagation paths such as illustrated in Fig. 6. But even with this impressive advance, the troubles were not over for the ray tracers. The propagation trajectories were now correct, but the methods for calculating them, usually based on $\vec{v}_{B}=\partial \omega / \partial \vec{k}$, the local dispersion relation, and Snell's Law, were terribly clumsy. Another twenty or more years were evidently to elapse before it was widely recognized by radiophysicists that the equation for the location of a wave packet,

$$
\frac{\vec{d}}{d t}=\vec{v}_{B}=\frac{\partial \omega}{\partial \vec{k}}(\vec{x}, \vec{k}, t)
$$

could simply be paired with its Hamiltonian companion

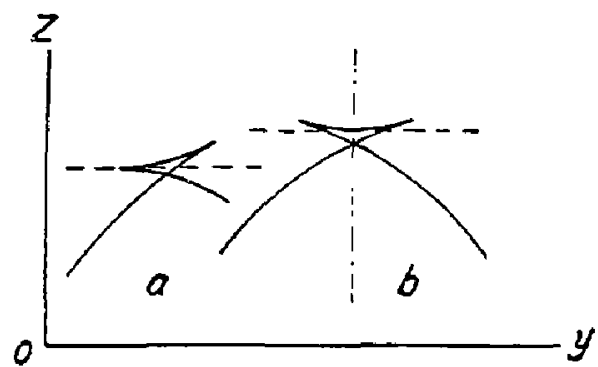

Fig. 5. Suggested propagation paths for radiowaves obliquely incident on a magnetized ionosphere. From Schekulin (1930).

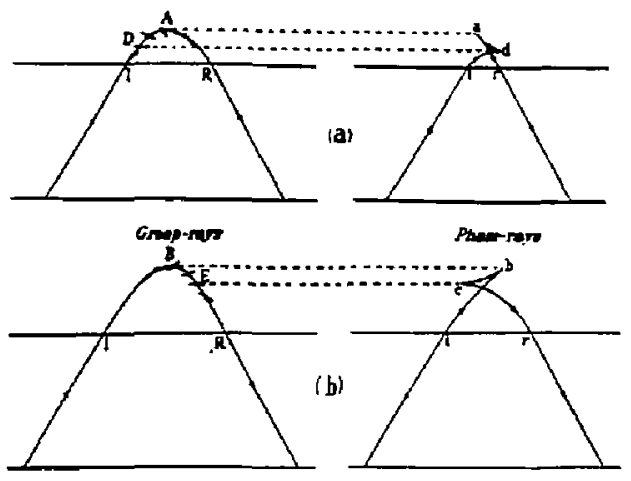

Fig. 6. Propagation paths based on group velocity (left) and phrase velccity (right) for radiowaves obliquely incident on a magnetized ionosphere. Note that the vertical components of the group and phase velocities are opposed over the D-A and B-E portio: is of the trajectory. The group velocity is horizontal at $a$ and $b$; phase velocity is horizontal at $d$ and e. From Booker (1938). 


$$
\overrightarrow{\mathbf{d}}=-\frac{\partial \omega}{\partial \overrightarrow{\mathbf{x}}}(\vec{x}, \vec{k}, t)
$$

in order to advance $\vec{k}$ in the trajectory calculation. ${ }^{7.9}$

\section{Ion Cyclotron Damping}

In addition to dispersion, another intriguing characteristic of plasma waves is collisionfree damping. As the name implies, this form of damping occurs for very long mean free paths, namely, $\lambda_{\mathrm{mfp}} \gg \lambda_{\text {wave }}$. Only a small number of particles in the distribution partake in the absorption of wave energy and the type of collisionfree damping depends on the resonant condition for these particles,
Landau damping,
Transit-time damping.
Cyclotron damping,
Cyclotron-hamonic damping,

$$
\begin{aligned}
& \omega-k_{\| v_{\|}}=0 \\
& \omega-k_{\|} \mathbf{v}_{\|}=0 \\
& \omega-k_{\| v_{\|}}-\omega_{c}=0 \\
& \omega-k_{\|} \mathbf{v}_{\|}-n \omega_{c}=0
\end{aligned}
$$

where $\omega_{0}$ is the cyclotron frequency, $q B / m c$, and $n$ is an integer. Landau and transit-time damping differ due to tine nature of the wave-particle coupling, being $q E$ and $\mu \nabla B$, respectively, in the two cases.

Although Landau damping was the first one to be given a theoretical description, 10 the first successfui experiments in this general area were those that demonstrated cyclotron damping. Figure 7 shows a 1958 ion cyclotron resonance heating (ICRH) experiment ${ }^{11}$ with plasma diagnostics based on rf coil impedance, 50-volt Doppler-broadening of C III impurity lines, and on d-d neutron production.

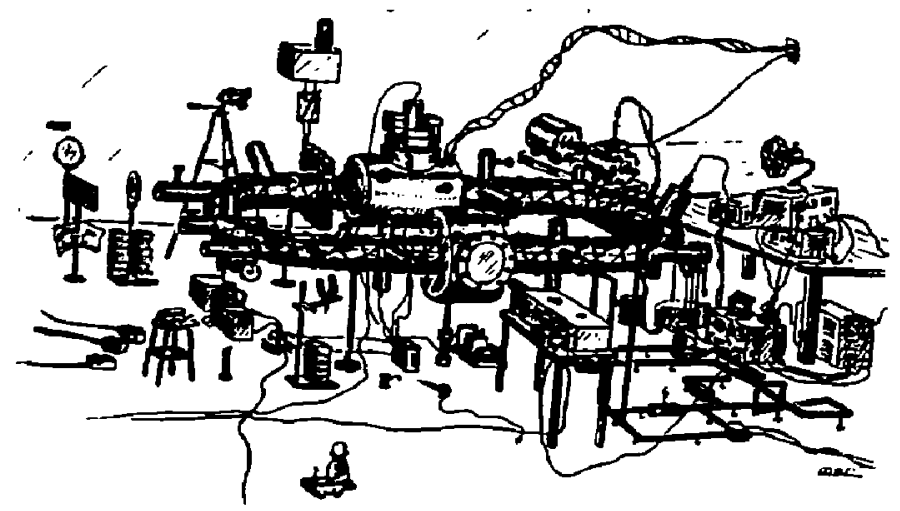

Fig. 7. Early sketch, by R.W. Palladino, of the B-64 stellarator. A neutron detector sits above the cylindrical shield box containing the 2-wavelength of induction coil (see Fig. 9). The nearer cylinder encloses the toroidal divertor. Also easily identifiable is the monochromator pointed into the divenor, the microwave waveguides, the helical windings and the fielderror correction apparatus (both shown quite schematically), the 400-cycle motor-generator fordischarge cleaning, and the ear muffs for suppressing the 400 -cycle howl. 
Data from this experiment, Fig. 8, shows increased radiation resistance for the coil at two frequencies, $\omega=\omega_{\mathrm{ci}}\left(B_{\mathrm{o}}=15090\right.$ gauss $)$ and at $\omega \equiv 0.91 \omega_{\mathrm{ci}}$. The latter frequency corresponded to the generation of ion cyclotron waves and produced heating of the total plasma, while exact cyclotron resonance led to deuteron acceleration underneath the coil and the production of $d-d$ neutrons.

The evidence for generation and damping in the 1958 experiment was indirect, but in 1961 a group led by $W$. M. Hooke ${ }^{12}$ demonstrated ion syclotron wave damping directly by magnetic probes placed in the plasma. Figure 9 gives a sketch of the apparatus, showing the profile of the magnetic field which decreased in magnitude on each side of the ICRH coil. Electromagnetic ion cyclotron waves generated under the coil, where $\omega<\omega_{c i}$, propagated into these two "magnetic beach" regions to be absorbed in the vicinity of the point where locally $\omega=\omega_{\text {ci }}(z)$. Figure 10 shows data from a magnetic probe moved axially through the plasma, giving the wave amplitude in the beach region for three values of $\omega / \omega_{\mathrm{ci}}(0)$, and confirms the strong attenuation of the wave near the $\omega=\omega_{c i}(z)$ point.

But an unexpected and fascinating thing happened when the machine field was set high enough so that the $\omega=\omega_{\mathrm{ci}}(z)$ condition was not satisfied anywhere between the mirrors. The wave amplitude increased going away from the coil.

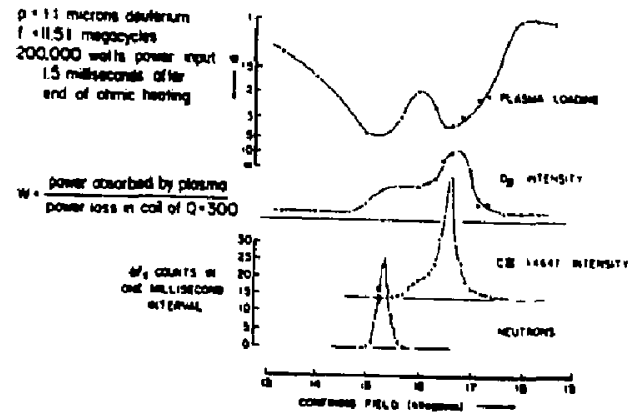

Fig. 8. ICRH plasma heating. From top to bottom: antenna radiation resistance; intensity of $D_{\beta}$ spectral line; intensity of $\lambda 4647 \mathrm{C}^{++}$spectral line; detected neutrons. From Stix and Palladino (1958).

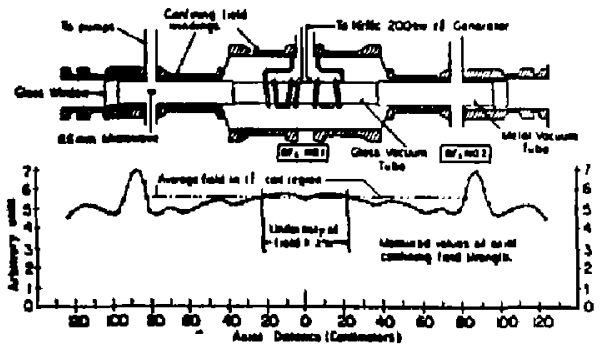

Fig. 9. Sketch of B-65 mirror-geometry ICRH experiment at PPPL. Note irregularly decreasing magnetic field ("magnetic beach") to left and right of if coil. From Hooke at al . (1961).

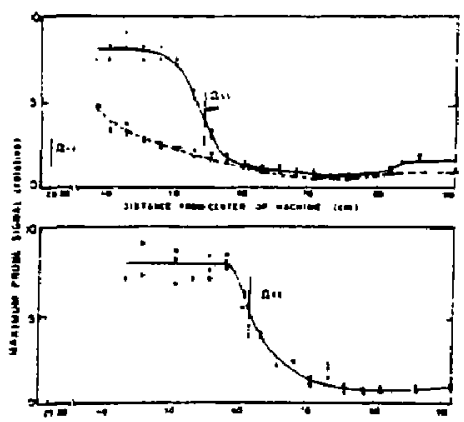

Fig. 10. Signals from a magnetic probe, measuring $\tilde{B}_{\mathrm{Z}}(\mathrm{z})$, in the magnetic-beach region of $\mathrm{B}-65$, for three different machine magnetic fields, $B d z), \quad \Omega=$ ! designates the point where $\omega=\omega_{\mathrm{t} j}(z)$ locally. From Hooke et al . (1961). 
Actually, as seen in Fig. 11, it went through a maximum, then a minimum, then a second maximum. Hooke's team was able to identify these wave-amplitude extrema with extrema in the local values of $\omega_{\mathrm{ci}}(z)$, the maximum wave amplitude appearing at the point of minimum $\omega_{c i}(z)$. WKB theory shows that $k_{k}(z)$ would also be a maximum where $\omega_{\text {ci }}(z)$ is a minimum, so even though the wave is not yet damped, it is slowed down, and conservation of the flux of wave energy then requires the undamped wave amplitude to increase. By analogy to wave behavior near a cutoff $\left(k_{\|} \rightarrow 0\right)$, the process found here now is given the generic title of "Airy swelling."

\section{Proton Whistlers}

The two waves discussed up to now are the whistler mode, which belongs to the fast-Alfvenwave branch of the dispersion curve, and ion cyclotron waves, which belong to the slow-Alfvenwave branch. In 1964 another totally unexpected event was observed - this time in data from the Alouette and Injun 3 satellites passing through the ionosphere. The team of Gurnett, Shawhan, Brice, and Smith ${ }^{13}$ identified spectrogram traces showing the coupling of these two branches, the conventional whistler triggering - at a location in the ionosphere where a certain critical condition is satisfied - the generation of an electromagnetic ion cyclotron wave, Fig. 12. Dispersion of the ion cyclotron wave as it traveled from its generation point to the satelite gave a whistling character also to its received signal. In this circumstance, the tone rises with time and asymptotically approaches the local iun cyclotron frequency. In

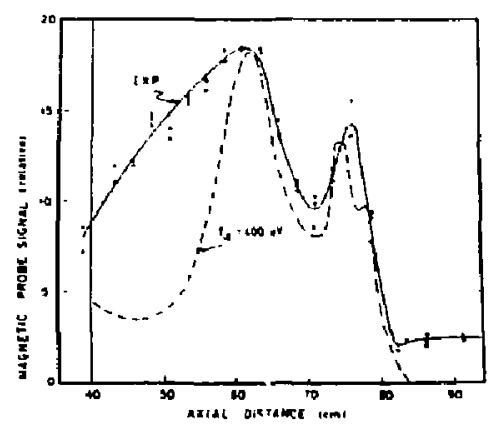

Fig. 11. Similar to Fig. 10, but with $B_{0}(x)$ such that $\omega<\omega_{\mathrm{ci}}(z)$ everywhere between the magnetic mirrors. From Hooke et al . (1961).
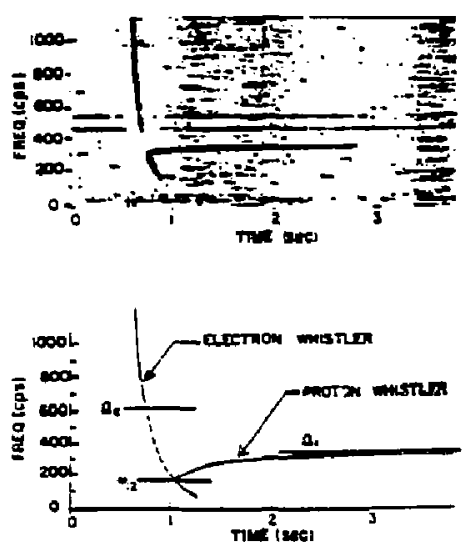

Fig. 12. Proton whistler signals observed in sateilite. data. Excited at a remote location by an electron whistler, the received proton whis!ler tone rises in frequency and asymptotically approaches the local proton gyrofrequency. From C. mett et al . (1965). 


\section{Page 8}

their classic 1965 paper, the authors note that the proton whistler was never observed without an electron whistler present, that the asymptotic proton whistler frequency was experimentally within $3.5 \%$ of the local proton gyrofrequency, and that the point of generation for the proton whistler could be identified with a critical plasma-parameter condition under which equations describing the two modes of propagation become strongly coupled. Moreover, the critical condition itself is unusual: the condition is that the plasma dielectric tensor element $\varepsilon_{x y}=0$, a condition that is never satisfied in a protonelectron plasma, but can only be satisfied if another ion species (e.g., $\mathrm{He}^{+}$in the ionosphere) is also present.

In satellite data a few years later, evidence was seen ${ }^{14}$ of absorption for at least the first six harmonics of the proton cyclotron frequency, Fig. 13. The physical basis for this absoiption process will be discussed in Section 6 , below.

In passing, it rilay be mentioned that it was to interpret data from the Alouette satellite that led Shkarofsky 15 to examine nelativistic effects on plasma waves. What is remarkable is that relativistic mass changes can be important even at very low temperatures, in order to obtain the correct dispersion relation near $k_{k}=0$ and $\omega \equiv n \omega_{c}$.

\section{Landau Damping}

1964, the year oi the proton whistler experiment, was also the year that Landau damping itself was given quansitative verification in laboratory experiments. Precise experiments were performed by Malmber's and Wharton ${ }^{16}$ on electrostatic electron plasma waves in a laboratory plasma with mean free paths about twenty times $(4000 \mathrm{~cm})$ the length of the apparatus. Accurate exponential decay in agreement with Landau theory was observed over two orders of magnitude of wave Fotver, and quantitative agreement was

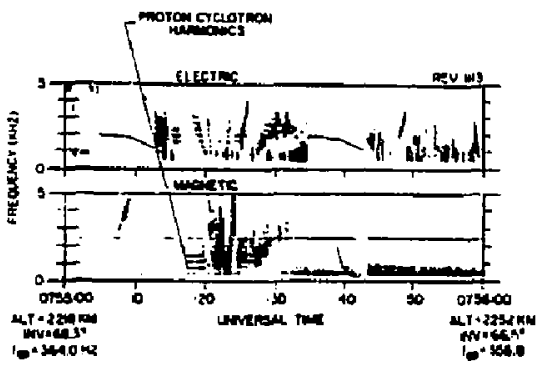

Fig. 13. Satellite data showing ion cyclotron harmonic structure on the electric and magnetic components of the received signals. From Gurnett et al . (1969).

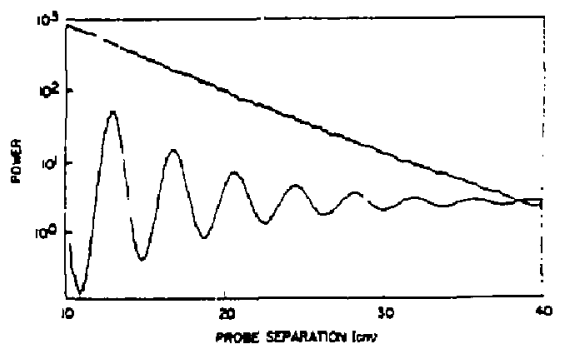

Fio. 14. Experimental observation of Landau damping of an electrostatic plasma wave Amplitude and interferometer signals plotted versus distance. From Malmberg and Wharton (1964). 
also found for the Langmuir-Tonks dispersion formula with the warm-plasina Vlasov-BohmGross correction. Figs. 14 and 15.

\section{Landau Undamping}

Up to this point, we have been discussing just one property of :ong mean-free-path plasinas collisionfree damping. Let us now look at another property: nonlocal response. By this one means that the response to a perturbing impulse can appear at a later time and at a different place from the original impulse. A long mean-free-path medium

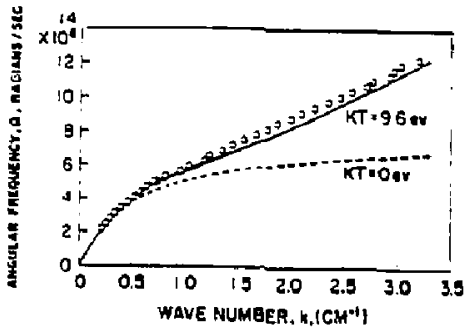

Fig. 15. Dispersion selation for electron plasma waves from experiment (points) and theory. An independent measure of electron temperature gave $k \mathrm{~T}_{\mathrm{e}}=9.6 \mathrm{eV}$. From Malmberg and Wharton (1966). has this property and its present local behavior can be influenced by the whole history of the plasma from earlier times and from other places. Collisionfres damping may be viewed in this framework. An initial disturbance may give rise to many modes, 17 but - since thu different modes move with different phase velocities -- the disturbance disappears with time as a result of destructive interference. On the other hand, under special conditions the modes may reassemble to produce a short-lived observable signal. In an elegant experiment, performed in 1968 on the same apparatus used for the Landau damping work, it was shown ${ }^{18}$ that - long after the disturbance had ostensibly disappeared by Landau damping - crherence cculd be reestablished. The reappearance, called a plasma wave "echo," can be explained qualitatively in a simple way. Consider that a grid, at $x=0$, is electrically modulated at frequency $\omega_{2}$. The modulations will produce perturbations to the plasma of the ballistic form, $f(x-y t)$, which might be written for this case

$$
f_{a}^{(1)}(x, v, t) \sim f_{a}(v) \exp \left[-i \omega_{a}\left(t-\frac{x}{y}\right)\right]
$$

Now a second grid, placed a distance $\&$ away, is excited at frequency $\omega_{\mathrm{b}}$. It also will produce firstorder perturbations, $f_{b}^{(1)}(x, v, t)-\tilde{f}_{b}(v) \exp \left(-i \omega_{b}[t-(x-l / v)]\right)$ but there will be a second-crder effect due to the modulation of the first group of electrons by the second grid,

$$
\begin{aligned}
f^{(2)}(x, v, l) & -\left(\frac{f_{b}^{(l)}(x, v, t)}{f_{b}(v)}\right) \\
& \sim f(v) \exp \left[-i\left(\omega_{a}-\omega_{b}\right) t+\frac{\omega_{a} x-\omega_{b} x}{v}+\frac{\omega_{b} \ell}{v}\right] .
\end{aligned}
$$

Integrating over all relocities, the velocity-dependent terms phase-mix the contributions to zero except where the velocity dependence in the exponent ciisappears, namely, where 


$$
x=\frac{\omega_{b} \ell}{\omega_{b}-\omega_{a}} .
$$

At this value of $x$, which can be far away from both grids, the phase mixing or Landau damping for the second-order perturbations is unscrambled, these perturbations all becoine coherent again and a marroscopic signal reappears. A plot of experimental data is given in Fig. 16.

\section{Finile-Larmor Radius and Crclotron-Harmonic}

\section{Effects}

Two more topics within the general area of long mean-free-path phencmena are finiteLarmor-radius and cyclotron-harmonic effects. These two topics are related because strong cyclotron-harmonic wave-particle interaction requires that the resonance condition, $\omega-\vec{k} \vec{v}=0$ be satisfied somewhere on the circular orbit. For harmonic resonance at $\omega=n \omega_{c}$, one then needs $k_{\perp} v_{\perp} \geq n \omega_{c}$, or $k_{\perp} \rho_{L}>n$.

As a simple illustration, one may consider the absorption of wave energy at the sacond harmonic of the cyclotron frequency $\left(\omega=2 \omega_{c}\right)$. Figure 17 shows the gyro-orbit of a particle in a wave field. When the particle is at position $A$, on the right hand side of the orbit, it is moving upiward, parallel to the weve field and absorbing energy at the rate $\vec{q} \cdot \vec{v}$. Half a period later, at position $B$, the particle is moving downward. The wave field, oscillating at twice the gyrofrequency. has now gone through a full time cycle. However, point $B$ is half a wavelength away from point $A$ and the direction of the $\vec{E}$ field at point $B$ is now downward. Again the particle absorbs energy from

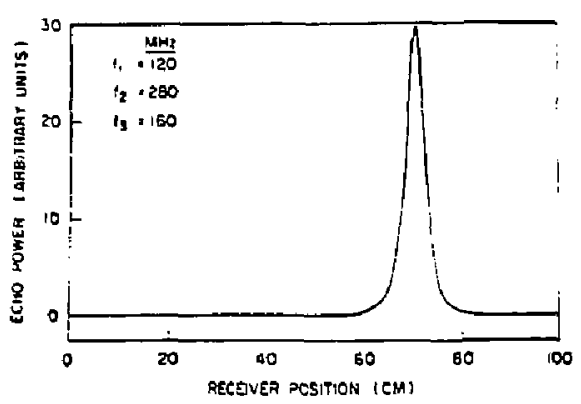

Fig. 16. Power at the echo frequency, $160 \mathrm{MHz}$, versus distance. The first grid, at $x * 0$, is excited at $120 \mathrm{MHz}$, and the second grid, at $x=40 \mathrm{~cm}$, at $280 \mathrm{MHz}$. The calculated position for the center of the echo $15 x=70 \mathrm{~cm}$. From Malinberg et al . (1968).
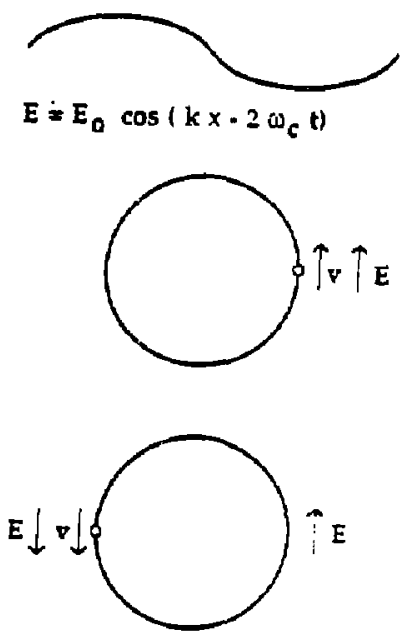

Fig. 17. Sketch of wave-particle interaction at $\omega=2 \omega_{\mathrm{c}}$. 
the field. As seen in the mechanism illustrated here, dbsoiption of wave energy at cyclotron harmonics depends striongly on finite-? aimor radius effects.

Fhase merwo $y$ is also important. Provided $k_{\perp} v_{\perp}>\omega$, there will typically be two points on the gro orbix witre the "Landau" resonance condition $\omega-\vec{k} \cdot \vec{v}=0$ is satisfied. As the gyrating particles pisses repeatedly through these resonare points, it beconies important whether they "remember" the phase associated with earlior passes. This point will be discused further in the following section.

While both ion and electron cyciotron hatmonic raciation had been observed in several experiments a few years earlier, by far the most dramatic and most influential experiment in this area was ti.at done by G. Landauer ${ }^{19}$ in 1961 , observing the noise emission from a simpit Penning discharge. The receiver frequency was $34.4 \mathrm{GHz}$, the magnetic field was varied between 2500 and 500 gauss, and all the harmonics between $\Omega=5$ and $n=$ 25 appeared, ricely separated, at his receiving antenna, Fig. 18. The kinetic implications of 5 is experiment were recognized by Canobbio and Croci, 20 namely, that the origin of the hamronic structure must be the excitation of electrostatic waves with extremely short wavelength, i.e., electron Bcrnstein waves with $k_{\perp}$ PLe $z n$. Then, in a related experiment in 1964, Buchsbaum and Hasegawa ${ }^{21}$ found that electron Pernstein modes could be established as radial standing waves inside a plasma column. It was to explain Landauer's original observation, in which if energy presumably in electron Bemstein running waves deep inside the plasma managed to propagate out to an external receiving antenna, that led to the aevelopment of mode-conversion theory, ${ }^{22} \mathrm{Fig}$. 19.

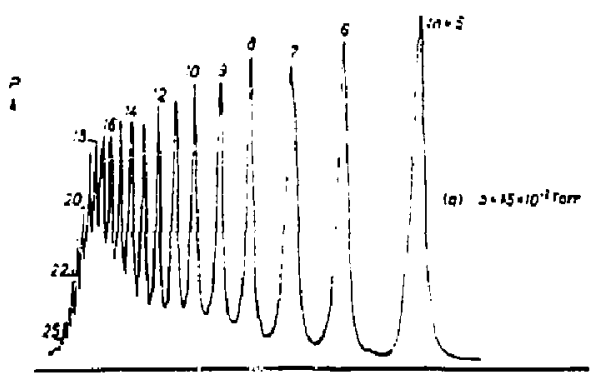

Fig. 18. Noise at $34.4 \mathrm{CHz}$ from Penning discharge. Horizontal axis is magnetic field; ticks at 500, 1000, 1500 , 2000, 2500, and 3000 gauss. From Landauer (1962).

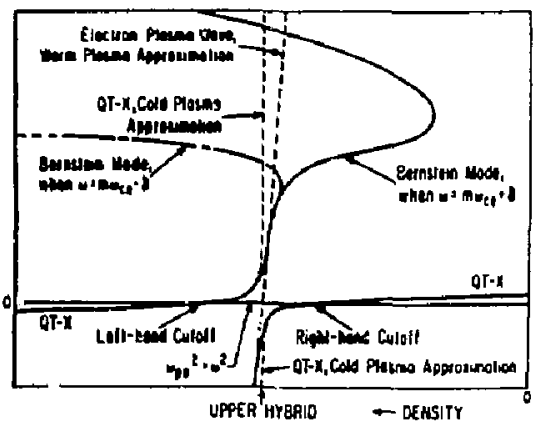

Fig. 19. Escape of electron Bernstein wave energy via mode conversion. Electrostatic Bemstein wäves, excited by nonMaxwellian electrons in a Penning discharge, travel first inward to higher dinsities. Their wavelengths become longer ( $n_{x}^{2}$ falls); finally the wave tunnels through to the QT-X branch and is able to escape the plasma as an alectromagnetic mode. From Stix (1965).

Mode conversion has been seen in many experiments in recent years. For example, work by Ono, Wong, and Wurden 23 have focused on ion Berristein wa:es. Mode conversion is again involved - these 
waves are launched as electrostatic electron plasma waves, $\vec{E} \| \vec{B}_{0}$, at the plasma edge, and modeconvert to $\vec{E} \perp \vec{B}_{0}$ ion-Bernstein waves beyond the lower-hybrid resonance layer $\left(\varepsilon_{x x}=0\right)$ inside the plasma. Some results from the ACT-I apparatus at Princeton are shown in Fig. 20. Another example, from very recent work on the Lausanne TCA tokamak, 24 is shown in Fig. 21 .

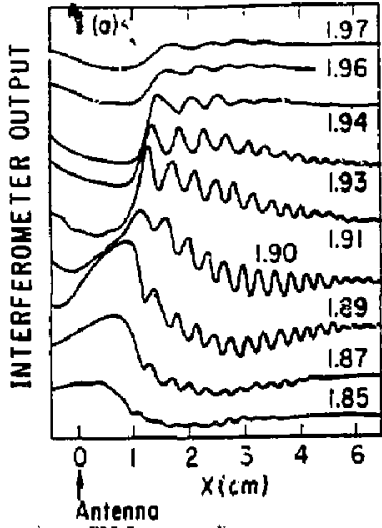

Fig. 20. Ion Bernstein wave data. Downstream interferometer traces for different values of $\omega / \omega_{c i}$ measured at $x=2 \mathrm{~cm}$, versus distance into the plasma. Electron plasma wave, at ieft, mode-transforms to an ion Bernstein waye at higher densities. From Ono, Wong. and Wurden (1983).

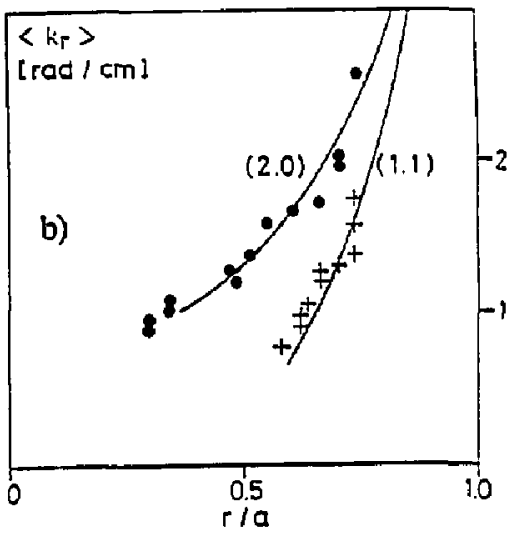

Fig. 21. Mode conversion of the shear Alfvén wave into the kinetic Alfven wave, from a 30 element infrared phase-contrast imaging diagnostic. Circles and crosses for $(n, m)$ mode structures of $(2,0)$ and $(1,1)$. Solid line is Chen-Hasegawa theory. 25 From H. Weisen et al. (1989).

\section{Large Values of $k_{\perp} \rho_{L}:$ Phase Mixing at Two Levels}

It was mentioned earlier that cyclotron-harmonic absorption is very much dependent on finiteLarmor-radius effects. For large values of $k_{\perp} \rho_{L}$, these effects can become dominant, and for wavelengths short compared to a Larmor radius, one may imagine that the effect of the magnetic field will actually disappear, at least for the ions. That is, the ion orbits might well be approximated uy straight-line trajectories. This conjecture turns out to be half true, and the correctic that has to be made to the straight-line orbit picture introduces an interesting new phenomenon, the occurrence of phase-mixing at two levels. To explain this comment, we first establish a basis for comparision, writing down the first-order distribution function, $\mathrm{f}_{1}$, for an unmagnetized plasma,

$$
f_{1}=-i \frac{q}{m}\left\{P\left(\frac{1}{\omega-\vec{k} \cdot \vec{v}}\right) \cdot i \pi \delta(\omega-\vec{k} \cdot \vec{v})\right] \vec{E} \cdot \frac{d f_{0}}{d \vec{v}}
$$

$P()$ indicates that the principal value is to be taken when integrating over $\vec{v} ;$ it is this part of $f_{1}$ that produces the reactive effe:f. $\delta()$ denotes the Dirac delta function, and this second portion of $f_{1}$, involving only the "resonant" particles, introduces Landau damping. 
Now for a magnetized plasma, in the limit of large $k_{\perp} \rho L$, it turns out that $f_{1}$ can be approximated by a quite similar expression, $26-29$

$$
f_{1}=-i \frac{q}{m}\left[P\left(\frac{1}{\omega-\vec{k} \cdot \vec{v}}\right)+\pi \cot \frac{\pi\left(\omega-k_{\|} \mid v \|\right)}{\omega_{c}} \delta(\omega-\vec{k} \cdot \vec{v})\right] \vec{E} \cdot \frac{d f_{0}}{\vec{d}} .
$$

The difference lies in that the $-i$, preceding the $\delta$ function in the expression for the unmagnetized plasina, has been replaced by a cotangent function. Now if we blur the gyroresonance by replacing $\omega$ by $\omega+i v$, and $i f v \geq \omega_{c^{\prime}}$ then the cotangent itself is about equal to $-i$ and the difference between the two $f_{1}$ 's disappears. On the other hand, if the imaginary part of $\omega$ is very small, then the phase of the cotangent term becomes purely reactive and there would appear to be no absorption. The exception occurs where the argument of the cotangent is an integral multiple of $\pi$, which occurs whenever

$$
\omega-\mathbf{k}_{\|} \mathbf{v}_{\|}=\mathbf{n} \omega_{c}, \quad \text { integer } \mathbf{n}
$$

that is, at cyclotron harmonic resonance. In this limit one may use

$$
\lim _{y \rightarrow 0^{+}} \cot (x+i y)=P(\cot x)-i \pi \delta[x \operatorname{modulo}(\pi)]
$$

and collisionfree absorption reappears, but qualified now by two delta functions, one specifying a Landau type resonance $(\omega \cdot \vec{k} \cdot \vec{v}=0)$ and the other, simultaneously, a cyclotron harmonic resonance, $\left(\omega-k_{\|} v_{\|}-n \omega_{c}=0\right)$. What happens here is phase mixing on two levels: on the first level, the familiar Landau resonance; at the second level, the destructive interference of the effects of phase-mixing on the first level except where the second criterion is simultaneously satisfied, namely, cyclotron-harmonic resonance. We tum now to look at some features of quasilinear theory, but will return to this question of phase mixing on two levels.

\section{Quasilinear Theory}

Quasilinear theory was introduced, in 1961, by theorists $30-32$ seeking to understand what limits the growth of linear-theory microinstamlities. They put forth the now familiar set of equations, for a one-dimensional unmagnetized plasms.

$$
\begin{aligned}
& \frac{\partial \mathrm{f}_{\mathrm{o}}(v, t)}{\partial t}=\frac{\partial}{\partial v} D_{\mathrm{v}} \frac{\partial \mathrm{f}_{\mathrm{o}}(v, t)}{\partial v} \\
& \frac{\partial}{\partial t}\left|E_{\mathrm{k}}\right|^{2}=2 \gamma_{k}\left|E_{k}\right|^{2}
\end{aligned}
$$

where $\gamma_{k}$ is the linear-theory growth rate for the $k^{\text {th }}$ Fourier mode. From second-order perturbation 
theory for the Vlasov equation, one obtains the velocity-space diffusion coefficient $D_{v}$.

$$
D_{v}=\lim _{L \rightarrow \infty} \frac{\pi q^{2}}{m^{2} L} \int_{-\infty}^{\infty} \mathbf{k} \mid E_{k} j^{2}\left[\delta\left(\omega_{k j}-k v\right)+r_{k}(\ldots)\right] .
$$

Although $D_{v}$ is derived from second-order perturbation theory, quasilinear theory immediately reincorporates $f_{2}$ into $f_{0}$. Then, instead of determining $\partial f_{2} / \partial t$ from a time-fixed $f_{0}(v)$, the equation involving $D_{v}$ becomes a diffusion equation for $f_{0}(v, t)$. Specifically, the equation describes the effect of quasilinear diffusion on the long-time-scale evolution of $\left.f_{0}{ }^{f} v, t\right)$.

Now in the case of the well-known "bump-on-tail" instability, the velocity-space diffusion described by $D_{v}$ flattens out the bump and thereby lowers the growth rate. Elucidating this point was a major initial success for quasilinear theory. In recent years, however, quasilinear theory has been applied not only is :istabilities, but - probably much more frequently - to the case of plasmas subject to intense rf excitation for the purposes of plasma heating and rf current drive. In this case $\omega_{k}$ is real, $\gamma_{k}=0$, and $D_{v}$ involves only the resonant particles, $i . e$. , the particles for which $\omega_{k r} \cdot k v=0$.

Applied to radiofrequency heating and to if current drive, the basic quasilinear equation is again that for the long-time-scale evolution of $f_{0}$. But a Fokker-Planck collision term is now added to the right side, so that the equation for the rf-accelerated particles reads

$$
\frac{\partial f_{0}(\vec{v}, t)}{\partial t}=Q f_{0}+C\left(f_{b}, f_{0}\right)
$$

where $Q$ is a quasilisear diffusion operator quadratic in the component: of the vector wave electric field and second order in the velocity derivatives. $C\left(f_{b}, f_{o}\right)$ is the Fokker-Planck Coulomb-collision term. For many purposes, it is adequate to approximate $\mathrm{C}\left(\mathrm{f}_{\mathrm{b}}, \mathrm{f}_{\mathrm{o}}\right)$ by the test-particle collisionaldiffusion operator in which $C\left(f_{b}, f_{0}\right)=C\left(f_{b}\right) f_{o}$, where the coefficients of the operator $C\left(f_{b}\right)$ depend only on the background distribution of ions and electrons, $f_{b}$.

For long if pulses, the distribution function approaches an asymptotic for $\mathrm{n}, \mathrm{f}_{\mathrm{o}}(\overrightarrow{\mathrm{v}})$, given simply by

$$
Q f_{0}+C\left(f_{b}, f_{0}\right)=0
$$

Applied to [CRF heating, the asymptotic $f_{D}(\vec{v})$ at low energies, $E<15 \kappa T_{b}$, is affected primarily by pitch-angle scattering against the background ions. $T_{b}$ is the background temperature of the ions and electrons. On the other hand, above $15 \kappa T_{b}$ it is mainly electron drag that affects the rf-accelerated 
ions. The effective temperature of the tail of the accelerated-ion distribution then depends only on $T_{e^{\prime}} n_{e}$ and on the ICRF power absorbed per unit volume, 33

${ }_{\mathrm{t}} \mathrm{T}_{\mathrm{eff}}=k T_{e} \cdot\left(1+\right.$ constant $\cdot$ ICRF Power $\left.\cdot \frac{T_{e} \mathrm{t} / 2}{n_{e}{ }^{2}}\right)$.

Figure 22 shows experimental data for $\mathrm{H}$ minority heating in a deuterium plasma in the PLT tokamak.34 The hydrogen charge exchange spectrum is shown, together with a theoretical curve based on quasilinear theory as described above, Eq. (19).

The long tail that is characteristic of ICRF heating can be used to enhance neutron production in a di-t plasma. Figure 23 shows a doubling of neutron production in the JET tokamak when ICRF was added to neutral-beam injection, 35 In the limit, it is estimated ${ }^{33}$ that one could, by this means, actually approach a nuclear power output equal to four times the if power input $(Q=4)$.

A similar analysis, balancing quasilinear of diffusion against collisions, may be made for $\mathrm{rf}$ current drive. In Fig. 24 contours of $f(\vec{v})$ are plotted in $v_{\perp}$, vil space for lower-hybrid current drive. 36

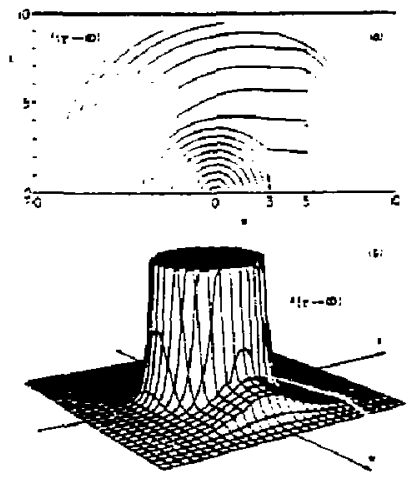

Fig. 24. Contours of $f(v)$ in $v_{1}, v_{\|}$space, for lower inybrid curzent drive. From Karney and Fisch (1979).

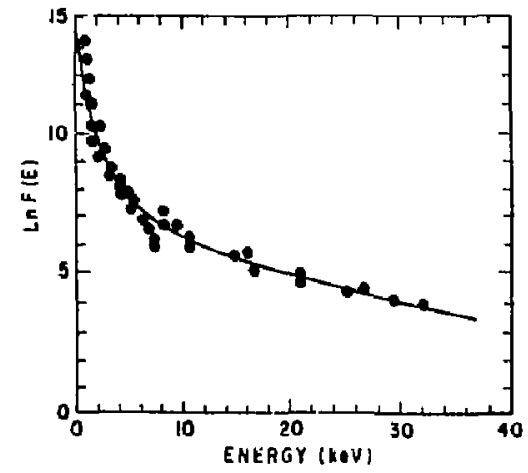

Fig. 22. PLT experimental data for hydrogen minority heating in a deuterium plasma. Hydrogen charge exchange spectrutn is shown, together with quasilineartheory curve fitted for $Z_{\text {eff }}$ and if power level. From Hosea et al . (1979).

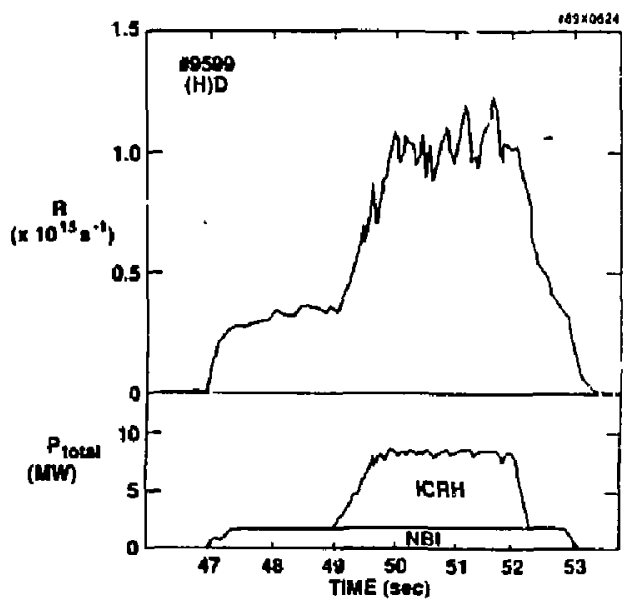

Fig. 23. DD reactions from JET when $\omega=\omega_{C D}$ ICRH was added to $D^{0} \rightarrow D^{+}$neutral-beam injection. From Cottrell et al. (1987). 
The determination of $\mathrm{f}_{\mathrm{e}}(\vec{v})$ is more difficult in the current-drive experiment than for $\hat{f}_{i}(\vec{v})$ in ICRH, but remarkably good agreement with quasilinear theory was obtained by Luce 37 by inverting the electron cyclotron emission spectrum, Fig. 25.

\section{Bounce-Geometry: Rabbit Ears}

Several interesting things come out of quasitinear theo:y when it is applied to radiofrequency power absorption by a plasma in a nonuniform magnetic field, such as that of a tokamak or a mirror machine. To begin with, in the nonuniform field, particles will pass through local regions of cyclotron resonance, picking up a kick in $v_{\perp}$ on each such pass. Both passing particles and trapped particles will undergo such acceleration. It turns out, however, that the inhomogeneity of the magnetic field actsally has very little effect on the total If power deposition. On the other hand, a small group of particles are strongly accelerated those whose mirroring points coincide with local cyclotron resonance, $\omega=\omega_{d}(r)$. These particles dwell a while near their turning point and, if that point is also one for if resonance, can pick up considerable energy. In addition, it can be shown that multiple passes through cyclotron resonance actually move the turning point and the if resonant point closer together. As a result, the velocitydistribution contours take on an unusual shape in $v_{\perp}, v_{\|}$space, developing "rabbit-ears" at the angle corresponding to those particles whose banana tips lie on an ti-resonant surface ${ }^{38}$ Figure 25 is a computer calculation 39 showing the rabbit ears stemming from rf resonance at the trajectory banana tips. The corresponding effect was seen in

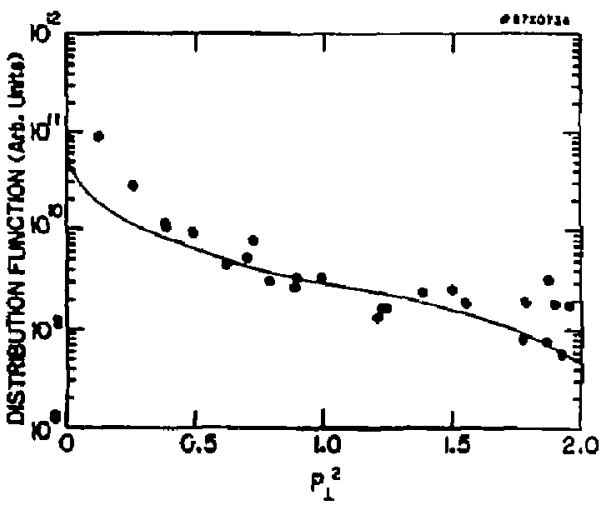

Fig. 25. $f\left(p_{\perp}\right)$ determined by inversion of electron syclotron emission at $90^{\circ}$ (points), compared to calculated quasilinear-theory distribution function for same experimental conditions for lower-hybrid current drive (curve). From T. C. Luce (1987).

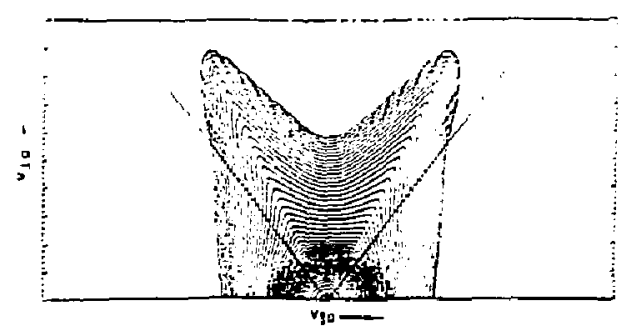

Fig. 26. Conto :s for steady-state ion distribution functions represented in tokamak mid-plane velocityspace coordinates. The angled lines denote the separation between trapped and passing particles, while the ends of the "rabbit ears" correspond to particles whose banana tips lie on the resonant surface. From Kerbel and McCoy (1985). 
experiments on $\mathrm{PLT}, 40 \mathrm{Fig}$. 27. Coming perhaps as no surprise once knowing of the occurrence of proton whistlers and proton cyclotron harmonic absorption in the Earth's ionosphere-magnetosphere system a ptienomenon that is apparently very closely related to these same rabbit ears has been abundantly observed in satellite data. Called "ion conjcs", the data show keV ions flowing upward into the magnetosphere, their distributions peaked in pitch angle and concentrated in a velocity-space cone. First observed by Sharp, Johnson, and Shelley 41 in 1977 , these events are now attributed, for the most part, to ion cyclotron, cyclotron harmonic or straight-line-trajectory lower-hybrid acceleration: the particle-wave interaction produces a major increment in $v_{\perp}$, which is followed by adiabatic upward streaming of the acceleraied icns along the Earth's magnetic field. changing their pitch angle. The conical structure in velocity space is clearly visible in Fig. 28. Original data from a mass-discriminating ion spectrometer located on a spinning satellite has been reduced $\mathbf{4 2}$ to familiar $v_{1}, v_{\|}$coordinates for the dominant species, $\mathrm{O}^{+}$.

\section{Bounce Averaging: Phase Mixing at Two Levels}

In the case of a nonuniform $\vec{B}_{0}$ field, the quasilinear equation is modified by drift kinetic theory,

$\frac{\partial f_{d}(E, \mu, t)}{\partial t}+v_{\|} \frac{\partial f_{0}(E, \mu, t)}{\partial s}=Q f_{0}+C f_{0}$

where $\left.v_{\|}= \pm[2 m(E-\mu B(s))]\right]^{1 / 2}$ and $s$ measures distance along $\vec{B}_{0}$. Note that $v_{\perp},{ }^{\prime \prime l l}$ have been replaced, as independent variables, by $E, \mu$, which

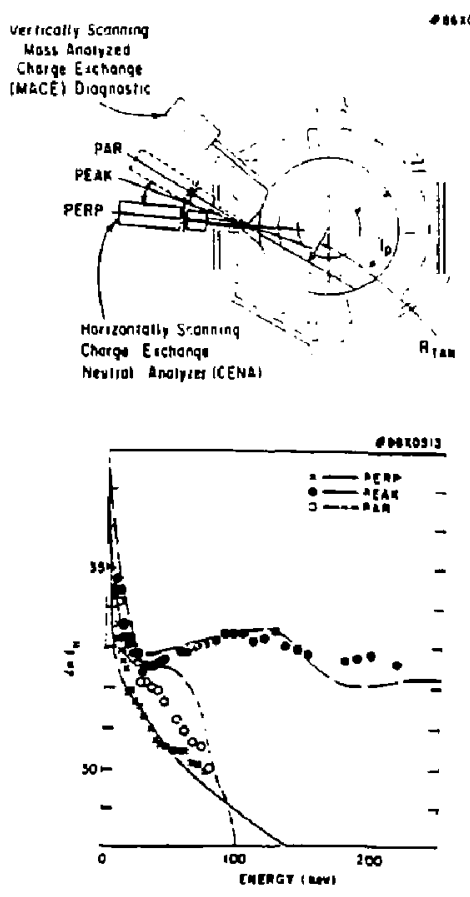

Fig. 27. Charge-exchange protr.n energy spectra during hydrogen minority ICRF heating on PLT. Detertor acceptance lines are drawn onto the top view of PLT, the one marked "Peak" leading to a remarkable high-energy" tail. The dashed lines are given by a numerical code based on bounce-averaged quasilinear theory. From Kaita, Hammett, and Wilson (1988).

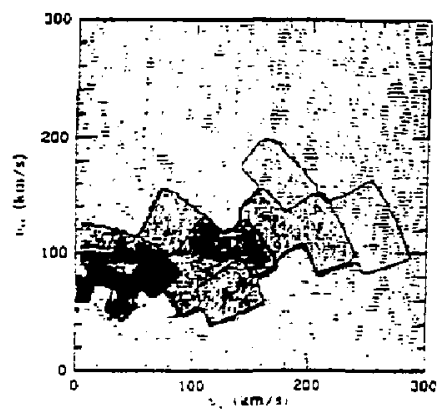

Fig. 28. Velocity distribution for energetic $\mathrm{O}^{+}{ }_{\mathrm{iG}} \mathrm{s}$ in the magnetosphere. Basecl on satellite-based ion spe:trometer data for an event on day 81288 . Darker regions correspond to greater phase-space density. Contours drawn at half decades. From Crewet al . (1989). 


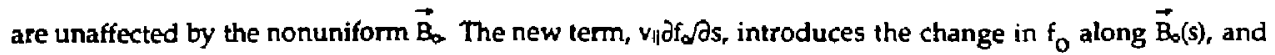
oscillates at the transit or bounce frequency. Bounce averaging annihilates this unpleasant term by taking the time average

$$
\left\langle\frac{\partial f_{o}}{\partial t}\right\rangle=\frac{1}{\tau_{b}} \int_{p} \frac{d s}{v_{\|}} \frac{\partial f_{o}(E, \mu, t)}{\partial t}=\frac{l}{\tau_{b}} \int_{p} \frac{d s}{v_{\| l}}\left(Q f_{o}+C f_{o}\right)
$$

where the integral, $\int_{p}$, extends over a transit or bounce period. $\tau_{b}$ is the bounce period. Now tpon evaluating the contribution to $<\partial f_{O} / \partial t>\operatorname{frcm} \int_{p}\left(d \alpha / v_{B}\right) Q f_{D}$, the phenomenon of phase mixing at two levels reappears. Assumir.g local rf-cyclotron resonance occurs at at least one point in the orbit, one finds, ignoring collisions, a result of the form 28

$\left(\frac{d f_{o}}{d \mathbf{d}}\right)-i \cot \left\{\frac{1}{2} \int_{p} \frac{d s}{v_{u}}\left[\omega-n \omega_{c}(s)\right]\right\}=i \cos \frac{x}{2}$

where the integral is over a transit or bounce period. If one blurs the phase memory from bounce to bounce by replacing $\omega$ by $\omega+i v$, and if $v t_{b}>1$, the cotangent goes to $-i$ and the absorption is blind to the bounce process. But for low collisionality, $v \tau_{b} \ll 1$, absorption occurs only for bounce resonance, that is, for

$\chi=\int_{p} \frac{s s}{v_{f l}}\left[\omega-n \omega_{c}(s)\right]=2 j \pi, \quad j$ integral.

Since $v_{\sharp 1}= \pm[2 m(E-\mu B(s))]^{1 / 2}$. the condition $\chi$ modulo $(2 \pi)$ will accur for a discrete set of $\mu$ values.

One may prepare a plot of $\mu$ (ordinatc) versus $\Sigma \chi$ ( abscissa) for a number of transits or bounces, where the value of $\mu$ is measured just after each transit and $\Sigma \chi$ is the accumulated phase difference also measured just after each transit. Fur zero amplitude of the ri field and for values of $\mu$ such that $j$ is integral, the plot will be a singli point, while for other values of $\mu$, the plot will be a horizontal line. But going to small but finite values
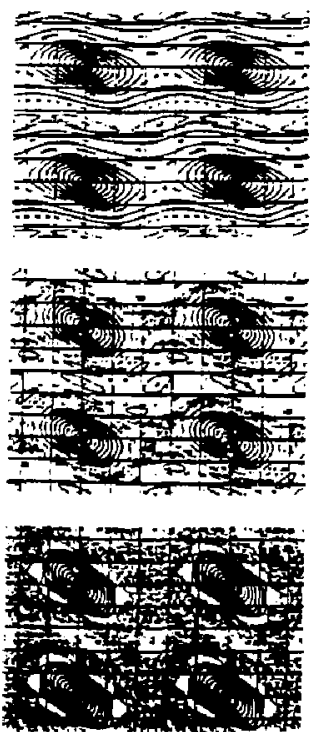

Fig. 29. Chirikov-Taylor Standard Map for three values $\therefore$ it the overlap (or stochasticity) parameter. Applied to uperadiabaticity in the case of cyclotron-frequency uxcitation of mirror-trapped particles, the ordinate is proportional to $\mu$ while the abscissa is $\Sigma \chi$ i.e., the accumulated change, mexsured after each transit, in the particle's gyrophase relative to the if phase. Two-level phase mixing corresponds to the case for a vanishingly small paragneter, i.e., vanishing island width. 
of the rf field, the plots change. The lines gentiy undulate but near the points they turn into islands, Fig. 29, top. The situation suddenly becomes familiar and one recognizes the surface-of-section plot associated with the Chirikov-Taylor Standard Map. For large amplitudes of the rf field, island overlap causes strong stochasticity, while weak if corresponds to adiabatic orbits almust everywhere. Our analysis of the two levels of phase mixing in linear wave theory corresponds to the weak of case. In the non-resonant instance, the changes to $\mu$ due to multiple passes through cyclotron resonance phase mix at the second-level and the accumulated effect remains small. The phase-space orbits show just mild undulations. But at resonance, a series of coherent increments accumulate to produce a significant modification to $\mu$ and islands appear in phase space. On the other hand, even at exact resonance, the maximum change in $\mu$ is limited to the island width and for this reason, the phenomenon - in the case where the if amplitude is finite ont most of the orbits are still adiabatic - has been termed superadiabaticity.

The context under which superadiabaticity was first examined ${ }^{43}$ was, in fact, just this situation of multiple passes through cyclotron resonance for mirror-trapped particles. But the effect is also seen in surface-of-section plots for cyclotron-harmonic acceleration, as discussed in the beginning of this section. Figure 30 plots $v_{\perp}$ versus gyrophase for ion motion at $\omega=30.23 \omega_{\text {ci }}$ with modest if amplitude. 44 The ordinate, $r$, in units of $k_{\perp} v_{\perp} / \omega_{C}$, ranges from 45 to 50 indicating that a Landau-type resonance, $\omega=30.23 \quad \omega_{c}=k_{\perp} v_{\perp} \cos (\vec{k}, \vec{v})$ will be satisfied at points satisfied along the helical

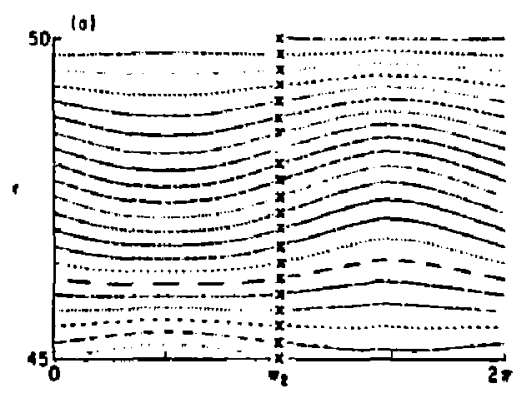

Fig. 30. Surface of sertion plot for cyclotron harmontic excitation. Ordinate, $r$, is in units of $k_{\perp} v_{1} / \omega_{c} ; \omega=30.23 \omega_{c}$. Modest if amplitude. Particles are followed for 300 transits; $x$ 's denote initial positions. From Karney (1978).
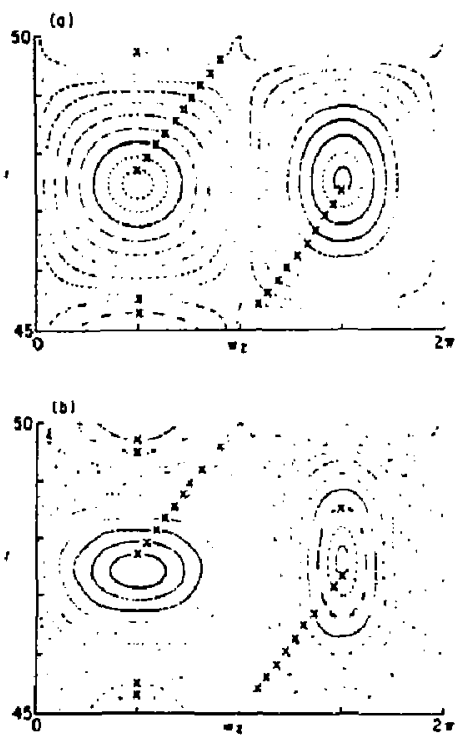

Fig. 31. Similar to Figure 28, but for $\omega=30 \omega_{c}$. Upper plot for modest if amplitude; lower plot for if amplitude that exceeds the stochastic threshold. 
trajectory. But because $\boldsymbol{r}\rangle$ is not an integral multiple of $\omega_{c}$, the velocity increments picked up from the Landau resonances phase mix, at the second level, to a small net sum. However, a very different story is told by irg. 31, where $\omega=30 \omega_{\mathrm{c}}$. At exact resonance, islands fill the entire space and the amplitude of the (weak) if field only affects the rate of movement along the contours. The situation is again superadiabatic because the excursions of $v_{\perp}$ are constrained to a single island. But at higher if amplitudes, stochasticity sets in and the trajectory can move along the stochastic web, achjeving large changes in $v_{\perp}$.

\section{RF Resonant Transpor:}

is a final topic, we turn to the question of rf-indured spazal diffusion. We saw earlier that the coincidence of banana tip and rf-resonance point could lead to the produstion of "rabbit-ears" on the ion distribution function, $f\left(v_{L}, v_{11}\right)$. The same circumsiance may also lead to actual loss of the strongly accelerated ions. In a tokamak, the canonical angular momeritim of $2 n$ ion is - by virtue of the toroidal symmetry - a constant,

$$
p_{0}=m v_{0}\left(R_{0}+r \cos \theta\right)+(q / c)\left(R_{0}+r \cos \theta\right) A_{0}=\text { constant }
$$

where $\vec{A}(\vec{r})$ is the vector potential. If the particle is given a small kick, the minor radius, $r$, of the flux surface on which the banana orbit is centered will change an amount $\delta$,

$$
\delta \mathbf{r}=\frac{\delta v_{\|}}{\Omega_{\theta}}=\frac{k_{\phi} \delta E}{m \omega \Omega_{\theta}}
$$

$\Omega_{\theta}$ the gyrofrequency in the poloidal magnetic field, and $\partial E$ is the change of particle energy due to rf acceleration. Since $\partial \mathrm{t}$ itself is proportional to $\partial E_{\text {, }}$ the $\mathrm{rf}$-induced transport is convective in nature ratier than diffusive. The full analysis of this problem $\mathbf{4 5}$ shows this convective radial flux, equal to $n_{t r a p}\langle\delta r / \delta t\rangle$, together with a quasilinear diffusive component of if transport.

A calculation of the effect of this type of wave-induced ion transport was made 46 for representative ICRF heating conditions in JET, Fig.

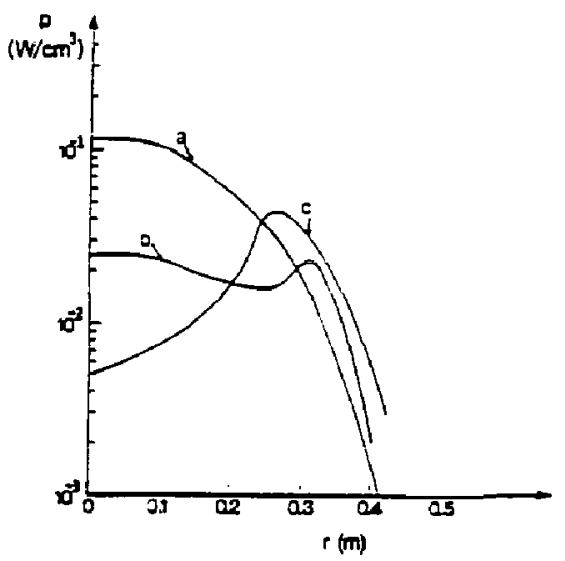

Fig. 32. For high-power ICRF heating in JET, the origina! if power absorption profile, $b$, is shown together with the ceposition profile, $c$, for power transferred from the accelerated minority ions to the bulk ions. Ir. the ausence of rf-induced transport, the two profiles would be the same, curve a. From T. Hellsten (1989). 
32. The original if power deposition in the JET plasma is limited in vertical extent by the focussing and configuration of the antenna; ri-induced outward transport then affects the time evolution of both the density profile of the rf-sesonant ions and the plasma temperature profile.

What appears to be experimental confirmation of this type of If transport has appeared in data obtained from particle detectors located at the outbuard edge of the TFTR plasma. ${ }^{47}$ Under conditions of hydrogen-minority ICRF fis: ting, an abundant flux of protons of $0.5-1.0 \mathrm{MeV}$ energy is seen, with an unexpectedly high rate at the detector positioned $45^{\circ}$ below the midplane, Fig. 33. Were the diffusion due to small-angle pitch-angle scattering of either passing or bananatrapped particles, one would expect the rate to peak at the midplane. The observed flux is very sensitive to the $r$ power level and to the location of the rf-resonanc: layer, relative to the detector position. A possible explanation for these observations is that these high energy particles received a rick of $\sim 100 \mathrm{keV}$ in a single pass through resonance.

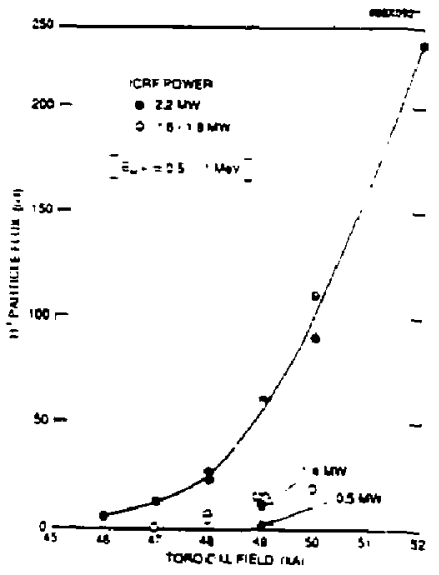

Fig. 33. Flux of protors escaping to Zweben's edge detector on TFTR during H-minority JCRF heating. An analysis of possible orbits suggests that these high energy particles received $-100 \mathrm{keV}$ in a single pass through resonance. From Colestocket al . (1989).

\section{Conclusion}

In conclusion, what has been presented has been a very limited selection of experiments together with their interpretation in the field of plasma waves. It is the author's hope that this quasihistorical review highlights the essential interplay not only between observation, theory, computer modelling and experiment, but also between the many disciplines that invoke plasma physics as a tool, including radio communication, astrophysics, controlled fusion, space physics, and basic research. 


\section{Acknowledgments}

It is a sincere pleasure to thank Drs. Herbert Berk, Abraham Bers, Tom Chang, Patrick Colestock, Philip Efthimion, Nathaniel Fisch, Gregory Hammett, Akira Hasegawa, Charles Karney. Charles Kennel, and Masayuki Ono for their help in assembling this presentation. This work was performed under United States Department of Energy Contract No. DE-AC02-76-CHO3073. 


\section{References}

'H. Barkhausen, 1.y.ys. Z. 20, 401 (1919).

${ }^{2}$ T.L. Eckersley, Nature 135, 104 (1935).

${ }^{3}$ E.T. Bu'rton and E.M. Boardman, Bell System Techn. J. 12, 498 (1933).

4L.R.O. Storey, Philos. Trans. R. Soc. London 246A, 113 (1953).

${ }^{5}$ L. Schekulin, Hochfrequenztech. u. Electroakust., 36, 172 (1930).

6H.G. Booker, Philos. Trans, R. Soc. London, 237A, 411 (1938).

${ }^{7}$ R.K. Luneburg, "Mathematical Theory of Optics", 1944 Lecture Notes, Brown Univ; republished in 1964 by Univ. California Press; also M. Kline and I.W Kay, "Electromagnetic Theory and ت̈eometrical Optics", Interscience, 1965.

6L.D. Landau and E. Lifshitz, "The Classical Theory of Fields", trans. by M. Hamermesh, AddisonWesley; 1951, p. 138.

${ }^{9}$ S. Weinberg, Phys. Rev. 126, 1899 (1962).

10L.D. Landau, J. Phy. (Moscow) 10, 25 (1946).

${ }^{11}$ T.H. Stix and R.W. Palladino, Proc. Second Intl. Conf. Geneva 31, 282 (1958).

${ }^{12}$ W.M. Hooke, F.H. Tenney, M.H. Brennan, H.M. Hill, Jr., and T.H. Stix, Phys. Fluids 4, 1131 (1961); also W.M. Hooke and M.A. Rothman, Nucl. Fusion 4, 33 (1964).

13D.A. Gurnett, S.D. Shawhan, N.M. Brice, and R.L. Smith, J. Geophys. Res. 70, $166^{\circ}$ (1965).

${ }^{14}$ D.A. Gurnett, G.W. Pfeiffer, R.R. Anderson, S.R. Mosier, and D.P. Cauffman, J. Geophys. Res. 74, 4631 (1969).

15I.P. Shkarofsky, Phys. Fluids 9, 561 (1966).

16].H. Malmberg and C.B. Wharton, Phys. Rev. Lett. 13, 184 (1964); also Phys. Rev. Lett. 17, 175 (1966).

17N.G. Van Kampen, Physica 21, 949 (1955). 18 J.H. Malmberg, C.B. Wharton, R.W. Gould, and T.M. O'Neil, Phys. Fluids 11, \147 (1968).

${ }^{19} \mathrm{G}$. Landauer, Proc. Fifth Intl. Conf. Ioniz. Phen. in Gases, Munich, 1, 389 (1961); also Plasma Phy. 4. 395 (1962).

20E. Canobbio and R. Croci, Proc. Sixth Intl. Conf. Ioniz. Phen. in Gases, Paris, 3, 269 (1963).

21S.J. Buchsbaum and A. Hasegawa, Phys. Rev. Lett. 12, 685 (1964).

22T.H. Stix, Phys. Rev. Lett. 15, 878 (1965).

23M. Ono, K.L. Wong, and G.A. Wurden, Phys. Fluids 26, 298 (1983).

${ }^{24}$ H. Weisen, K. Appert, G.G. Borg, B. Joye, A.J. Knight, J.B. Lister, and J. Vaclavik, Phys. Rev. Lett. 63,2476 (1989).

25 A. Hasegawa and L. Chen, Phys. Rev. Lett. 35, 370 (1975).

26D.E. Baldwin and G. Rowlands, Phys. Fluids 9, 2444 (1966). 
27R.E. Aamodt, Plasma Phys. 9 , 1573 (1967).

28H.L. Berk and D.L. Book, Phys. Fuids 12, 649 (1969).

${ }^{29}$ B. Coppi, M.N. Rosenbluth, and R.N. Sudan, Anr. Phys., (N.Y) 55, 207 (1969).

30w.E. Drumunand and D. Pines, Nud. Fusion Supp. Pt. 3, 1049 (1962).

$3 \mathrm{i}$ A.A. Vedenov, E.P. Velikhov, and R.Z. Sagdeev, Nucl. Fusion 1, 82 (1961); Nucl. Fusion Supp. Pt. 2, 423 (1962).

32Yu. A. Romanov and G.F. Filippov, Sov. Phys. JETT 13, 87 (1961).

${ }^{33}$ T.H. Stix, Nucl. Fusion 15, 737 (1975).

34 J. Hosea, S. Bernabei, P. Colestock, S.L. Davis, P. Efthimion, R J. Goldston, D. Hwang, S.S. Medley, D. Mueller, J. Strachan and H. Thompson, Phys. Rev. Lett. 43, 1802 (1979).

35G.A. Cottrell, V.P. Blıatnagar, J.G. Cordey, W. Core, S. Corti, H. Hamnén, T. Hellsten, J. Jacquinot, F. Sand, D.F.H. Start and M. Watkins, in AIP Conf. Proc. 159, "Applications of RF Power in Plasma," Seventh Topical Conference, Kissammee, FL 1987, ed. by S. Bernabei and R. W. Motley, p. 290.

${ }^{36}$ C.F.F. Karney and N.J. Fisch, Phys. Fluids 22, 1817 (1979).

${ }^{37}$ T.C. Lure, "Superthermal Electron Distribution Measurements with Electron Cyclotron Emission", Ph.D. Thesis, Princeton University (1987).

38T.D. Rognlien and Y. Matsuda, Nucl. Fusion 21, 345 (1981).

39 G.D. Kerbel and M.G. McCoy, Phys. Fluids 28, 3629 (1985).

${ }^{40}$ G.W. Hammett, R. Kaita, and J.R. Wilson, Nucl. Fusion 28, 2027 (1988).

${ }^{41}$ R.D. Sharp, R.G. Johnson, and E.G. Shelley, J. Geophys. Res. 82, 3324 (1977).

${ }^{42}$ G.B. Crew, T. Chang, J.M. Retterer, W.K. Peterson, D.A. Gumett, and R.L. Fiuff, J. Geofhys. Res, to be published. (Note to editor. The manuscript was accepted for publication in JGR on 10/13/89.) Contains 61 references.

${ }^{43}$ M.N. Rosenbluth, Phys. Rev. Lett. 29, 408 (1972).

${ }^{44}$ C.F.F. Kamey, Phys. Fluids 21, 1584 (1978).

45L. Chen, J. Vaclavik, and G.W. Hammett, Nucl. Fusion 28, 389 (1988).

${ }^{46}$ T. Hellsten, Plasma Physics and Controlled Fusion, 31, 1391 (1989).

47P. Colestock, A. Cavallo, W. Dorland, J. Hosea, G. Greene, G. Hammett, H.W. Hendel, B. Howell, K. Jaehnig. R. Kaita, S.S. Medley, C.K. ''hillips, A.L. Roquemore, G. Schilling, J. Stevens, B. Stratton, D. Smithe, A. Ramsey, G. Taylor, J.R. Wilson, S.J. Zweben, W. Gardner and D. Hoffman, in AJP Conf. Proc. 190, "Radio-Frequency Power in Plasmas", Eighth Topical Conference, Irvine, CA 1989, ed. by R. McWilliams, p. 189. 


\title{
NOTICE
}

\author{
Available from:
}

National Technical Information Service

U.S. Department of Commerce

5285 Port Royal Road

Springfiele Virginia 22161

703-487-4650

Use the following price cotes when ordering:

\section{Price: Printed Copy A03 Microfiche A01}

\section{DISCLAIMER}

This report was prepated as an account of work sponsured by an agency of the United States Government. Neither the United States Government nor any ageney thereof, nor any of their employees, makes any warrant; express or implied, or assumes any legal liability of respotaibility for the accuracy, completeness, or usefulness of any information, apparatus. preduct, of process disclosed, of represents that its use would not infringe privalely owned -ights. Re:-e. ence herein to any specific commercial product, $p_{i}$-ness, or service by tracie tame, Irad . .... manulacturer, or atierwise does not necessarily constitute or imply its enjorsement. ommendation, or favoring by the United States Government or any agency thereof. The views and opinions of authors expressed herein do not rocessarily slate of reflect those of the United States Government or any agency thereff. 